International Journal of Modern Physics: Conference Series

(C) World Scientific Publishing Company

\title{
PULSAR WIND NEBULAE MODELING
}

\author{
NICCOLÒ BUCCIANTINI \\ INAF Osservatorio Astrofisico di Arcetri \\ L.go Fermi 5, Firenze, FI, 50125, Italy \\ niccolo@arcetri.astro.it
}

Received Day Month Year

Revised Day Month Year

\begin{abstract}
Pulsar Wind Nebulae (PWNe) are ideal astrophysical laboratories where high energy relativistic phenomena can be investigated. They are close, well resolved in our observations, and the knowledge derived in their study has a strong impact in many other fields, from AGNs to GRBs. Yet there are still unresolved issues, that prevent us from a full clear understanding of these objects. The lucky combination of high resolution $\mathrm{X}$-ray imaging and numerical codes to handle the outflow and dynamical properties of relativistic MHD, has opened a new avenue of investigation that has lead to interesting progresses in the last years. Despite all of this, we do not understand yet how particles are accelerated, and the functioning of the pulsar wind and pulsar magnetosphere, that power PWNe. I will review what is now commonly known as the MHD paradigm, and in particular I will focus on various approaches that have been and are currently used to model these systems. For each I will highlight its advantages, limitations, and degree of applicability.
\end{abstract}

Keywords: star: pulsar; magnetohydrodynamics; relativity; ISM: supernova remnants

PACS numbers: $98.38 . \mathrm{Mz}, 97.60 . \mathrm{Gb}$

\section{Introduction}

Pulsar Wind Nebulae (PWNe) are bubbles of relativistic particles, pairs and perhaps ions. They are due to the interaction of the ultra-relativistic wind from a pulsar either with the parent Supernova Remnant (SNR) or the $\operatorname{ISM}^{1,2,3}$. They emit a broad band spectrum extending from radio to $\gamma$-rays, via synchrotron and Inverse Compton emission ${ }^{4,5,6,7,8}$. Among all PWNe, the Crab Nebula ${ }^{9}$ takes a special place. It is such a well known system, that its properties are a benchmark for our models.

To model a PWN one needs an acceleration mechanism responsible for producing the non-thermal particle's distribution function that is observed, and a dynamical model describing the advection and evolution of the flow.

To these days the acceleration problem remains unsolved. Ions in the pulsar wind, carrying a large fraction of the total wind energy, could accelerate pairs, but there is no compelling evidence for their presence ${ }^{10,11}$. Efficient Fermi-like acceleration of a pure pair plasma, requires a wind with an eccessively small 
magnetization $^{12}$. Reconnection of a striped wind ${ }^{13,14,15}$, leads to hard spectra, as the ones observed in radio, but requires winds with properties not compatible with standard pulsar wind dynamics ${ }^{16,17,18}$. There is also the possibility of continuous distributed turbulent acceleration in the bulk of the nebula ${ }^{19,20}$. There is however a general consensus that X-ray emitting particles are accelerated at the wind termination shock. The origin of radio emitting ones is still currently debated ${ }^{21}$.

This contrasts with dynamical models for the flow properties that have been developed to a greater success. They are based now on a MHD paradigm. In fact the gyro-radius of the particles is much smaller that the size of these nebulae, even up to the highest observed energies. A fluid description is thus possible, and can be formulated in terms of MHD. To build an emission model, one just needs to couple it with a prescription for particle's acceleration. The hope is to reproduce the observed morphology, to relate it to the pulsar wind properties, and to provide a unifying picture for all these objects.

Recently, the tools and approches that have been used in the investigation of PWNe have been applied to Long and Short GRBs ${ }^{22,23,24,25}$. Indeed these systems too can be modeled as expanding relativistic magnetized bubbles, and share a large amount of physics and dynamics with PWNe.

I will review in the following the various approaches that have been presented in the literature to model these systems, discussing their pros and cons, in relation to the questions one would like to answer.

\section{1-Zone Models}

We call 1-Zone model any model where the PWN is described as an uniform object, with a given size (radius) possibly time-dependent. The internal structure is completely neglected. These models, notwistanding their simplicity, have proved very successful for the investigation of the global average properties of PWNe. In particular their interaction with the partent SNR. The first 1-Zone model for PWNe was developed by Ref. 26, with application to the Crab nebula. They studied the evolution of particles in an expanding bubble, subject to adiabatic losses, radiation losses and a continuous injection. The analytical approach they adopted is however justifiable only for young systems in the so called free-expansion phase (the first few thousands years). Subsequently this model has been extended to take partially into account the interaction with the $\mathrm{SNR}^{27,28}$, or changes in injection history ${ }^{29}$. Only recently a numerical approach was been used $30,31,32,33,34,35,20$. This allows us to follow the evolution for a longer time and to take into account the various phases of the interaction with the parent SNR. Moreover we can model many different distribution functions, that are not necessarily a single power law (either broken power laws have been considered, or a power-law plus a Maxwellian). In particular, the work by Ref. 35 is exemplary for showing the richness of behaviors that is found and the degree of complexity that one can investigate using these simple tools.

1-Zone models are quite easy to build and they allow us to sample a large pa- 
rameter space of often unknown injection or evolutionary properties. The Radio emission properties, the location of spectral breaks, the ratio of synchrotron to Inverse Compton emission, can be fairly well modelled. Of course one should not expect more than a rough quantitative agreement with the global observed properties of these systems, and they are not reliable for the high-energy X-ray properties, that depend on the post shock flow. They are also quite good in recovering the duration and timescale of the various phases of interaction with the SNR.

Unfortunately, quite often the quality of the available data is limited, and 1-Zone models are then the only justifiable approach. They also constitute a good starting point for more accurate, multidimensional investigations.

\section{1-D Models}

It is a general expectation that, increasing the dimensionality of a model, should lead to a more realistic description of a system: a description closer to the true working. Including a dependence on the radial coordinate from the pulsar leads to 1-D models. The first 1-D model of the Crab Nebula was presented by Ref. 36 . Several spatial features could be explained.

- the under-luminous region, centered on the location of the pulsar, and interpreted as the ultra-relativistic unshocked wind.

- the wavelength dependent size of the Crab Nebula, with radio emission extending beyond the X-ray one, explained in term of combined bulk flow from the termination shock to the outer edge of the nebula, and synchrotron cooling.

- the existence of a bright torus, understood as due to compression of the magnetic field in the outer layers of the nebula.

This was also the fist time nebular properties were used to infer the Lorentz factor and/or magnetization of the wind. This model has been further developed by Ref. 37, 38 who solved the full set of relativistic MHD equations, and provided a quantitative estimate of the structural properties of the Crab Nebula. Later Ref. 39 presented a time dependent analytical solution.

More recently numerical investigations have allowed us to avoid many of the simplifications required by the early analytical treatment. Ref. 40 and Ref. 41 were the first to investigate the dynamics of the PWN-SNR interaction using a hydrodynamical code, for all the various evolutionary phases: the free-expansion phase ${ }^{42}$, the reverberation phase, and the final Sedov phase. Similar works have also been carried out by Ref. 43, 44 which were the first to use relativistic MHD and to investigate the role of magnetic field. More recently Ref. 45 have used a 1-D model to compute the evolution of an injected distribution function, and to compute an integrated spectrum for G21.5-0.9.

Out of 1-D models was born the so called $\sigma$ problem $^{46}$. The $\sigma$ problem is twofaced: one related to the efficiency of accelerating particles at a shock, the other to 
the ability of slowing down a relativistic magnetized outflow. Here I will only consider this latter aspect. In Ideal MHD, a radial highly-magnetized (with a toroidal field) supersonic relativistic pulsar wind cannot be slowed down by a shock to match the typical expansion speeds of the confining SNR. It only happens for a small value $^{37}$ of the magnetization $\sim 10^{-3}$. This contrasts strongly the expectations of pulsar wind electrodynamics ${ }^{47,48,49,50}$, which suggest that the magnetization in the wind, at best, with minor deviations from radial outflow, could be $\sim 1$ at the typical distance of the nebulae ${ }^{51}$.

Moreover 1-D models fail to explain the relative size of X-ray an Radio in the Crab Nebula ${ }^{52}$, and the correct softening of the spectrum with the distance from the pulsar ${ }^{53}$. These problems are related to the fact that in 1-D models there is a one-to-one correspondence between distance, age, magnetic field and flow velocity. This inevitably leads to onion-like structures, which are the exact opposite of the complete mixing at the base of 1-Zone models.

\section{2-D Models}

The interest in multidimensional models for PWNe grew mostly in the past ten years, and was mainly driven by optical and X-ray images from HST, CHANDRA and XMM-Newton. High resolution images have shown that the inner region of PWNe is characterized by a complex axisymmetric structure, generally referred as jet-torus structure. It was observed at first in the Crab Nebula ${ }^{54,55}$, and it has since been detected in many other PWNe $\mathrm{PW}^{56,57,58,59,60,61,62}$. Today there is a general consensus that such structure is always present in young nebulae.

It is evident that, in the presence of a toroidal magnetic field, anisotropic stresses will arise in the nebula, defining a preferential symmetry axis. Models that assume a cylindrical geometry are referred as 2-D models. Ref. 63 were the first to develop a 2-D model accounting for magnetic tension, in an attempt to explain the observed prolate geometry of the Crab Nebula, and to use it as a further constrain on the plasma magnetization.

Given the complex dynamics, associated with the larger degrees of freedom due to the increased dimensionality, in order to move beyond the simple analytical solution by Ref. 63 , we had to wait for numerical codes for computational fluid dynamics. ${ }^{64,65}$ Ref. 66 was the first to present a numerical model of a prolate nebula applied to 3C58. It was however the investigation of the dynamics in the vicinity of the termination shock that drove most of the successive attempts at modeling the inner jet-torus structure.

At the typical distance of the termination shock, the wind luminosity and magnetization are not uniform, but can be described by the so called split monopole solution: most of the energy is confined to the equatorial region, and there is no evidence of jets or collimated pulsar outflows along the axis. This is confirmed both by theoretical ${ }^{67,49}$ and numerical ${ }^{68,50,69,70,71,72}$ studies. Such a wind would naturally drive a complex dynamics at least in the inner region. Ref. 73,74 were the 
first to investigate this problem, modeling the flow dynamics at the termination shock. Later Ref. 75 suggested that hoop stresses in the body of the nebula could lead to the collimation of the jet. This has been extensively confirmed numerically by different groups ${ }^{76,77,78,79,80}$. The results of this numerical investigation went far beyond the confirmation of these simple ideas. Many aspects of the nebular morphology have been investigated: the details of emission maps ${ }^{79}$, polarization ${ }^{81}$, the integrated broad-band spectrum ${ }^{80}$. We can now reproduce reasonably well many of the observed X-ray features of the Crab Nebula, including the wisps and the knot, in terms of relative size and brightness. Recently the same tools have been applied to the modeling of variability. PWNe have been known to be variable in the X-rays since the first observations, in particular in the wisps region ${ }^{54,82}$. Such variability can be reproduced by 2 -D models ${ }^{83,3,84}$ both in term of its typical timescale and in its morphological pattern, of outgoing waves.

Concerning the wind magnetization $\sigma, 2$-D models have shown that values $\sim$ $0.03-0.1$ are required to explain the observed morphology and the presence of a jet; about one order of magnitude higher than in 1-D models. This for two reasons: the extra degree of freedom of the 2-D geometry allows to accommodate more magnetized winds; a large degree of internal fluid turbulence and mixing. This strongly suggest that reality could be closer to the full mixing assumption of 1-Zone models, instead of the onion-like structure of 1-D models.

Mostly developed to investigate the dynamics of the internal region, 2-D models have been also applied to the evolution of the PWN-SNR system. Ref. 40 were the first to investigate the 2-D dynamics of the reverberation phase, showing the high level of mixing between the SNR and PWN, and the role of density gradients in the ISM, leading to a relic PWN displaced with respect to the pulsar, as it is often observed in old systems. Ref. 85 and Ref. 86 were the first to investigate the Rayleigh-Taylor instability at the interface with the SNR ejecta, both in the magnetized an unmagnetized case, showing the development of the filamentary network observed in the Crab Nebula. Ref. 87 investigated in detail the interaction of a pulsar moving across the SNR, the transition from spherical PWNe in the early phase to a cometary structure, and in Ref. 88 the interaction with the SNR forward shock was also taken into account. Recently Ref. 89 analyzed the role of ISM density gradients, in the presence of an ordered magnetic field.

\section{3-D Models}

It has been known for a long time $\mathrm{e}^{90,91}$ that 2 -D configurations with a purely toroidal magnetic field, in 3-D are subject to current driven instability (kink instability). Instability of the jet is seen in $\mathrm{PWNe}^{59,92}$. To fully capture the dynamics associated with this instability full $3-D$ models are necessary. Only recently this process has been studied with numerical codes in the simplified regime of a magnetized column confined by a hot atmosphere $\mathrm{e}^{93,94,95}$. The instability reaches the non-linear regime on a typical Alfvènic timescale, and after about 10 Alfvènic timescales, the original 
toroidal field has almost completely vanished. This process could easily solve the $\sigma$ problem $^{90}$, but one must be carefull because the high level of polarization ${ }^{96,97,98}$ suggests that this instability cannot be so violent. Part of the reason for this inconsistency can be found in the simplified regime that these models consider. In PWNe the toroidal magnetic field is continuously injected by the pulsar wind, and the PWN is not pressure confined but confined by a high density wall at the contact discontinuity with the SNR ejecta. This implies that there must be a balance between the injection and dissipation of the toroidal field. Moreover in the inner region, close to the termination shock, where the plasma is injected, the magnetic field is bound to be close to toroidal, because instabilities would preferentially act in the outer regions. Unfortunately a full 3-D simulation of a PWN, evolving long enough to reach a balance, has not been carried out yet, mostly because of computational cost.

However recently Ref. 99 (see also these proceedings for a more detailed discussion) presented a very interesting preliminary study, where the dynamics of the PWN in a full 3-D setting was investigated for a time-length corresponding to 70 yr. While this is about one order of magnitude smaller than the age of the Crab Nebula, and it can be debated if the nebula has reached a state of dynamical equilibrium, nevertheless this timescale is longer that a typical Alfvènic crossing time, and longer than the synchrotron cooling time for X-ray emitting particles. The results confirm the qualitative expectations: the inner region close to the termination shock is still dominated by a toroidal field, and preserves the axisymmetric structure of 2D simulations; a jet is formed and extends in the body of the nebula; instabilities at the outer edge of the nebula act to reduce the net toroidal field component. It is also found that the nebula can accommodate even a wind with high $\sigma$, of order unity. Recalling that, even minor deviations of the wind from a purely radial flow, can lower $\sigma$ to values of order unity, at the typical distance of the termination shock radius $^{51}$, this suggests that the $\sigma$ problem is likely an artifact of the 1-D geometry of theories of old. However this does not rule out the possibility of other non-ideal effects, like dissipation and/or reconnection to play a role. It also does not solve the $\sigma$ problem from the point of view of particle's acceleration. But it suggests that one could seek for independent solutions of these two aspects, instead of looking for one that solves both at once.

\section{Bow-Shocks}

Despite the fact that few works have been devoted to the multi-dimensional investigation of the dynamical evolution of old PWNe inside SNRs, there are several works focusing on the interaction with the ISM. Pulsars moving in the ISM produce the so called pulsar wind bow-shock nebulae $e^{100,101,102,103,104,105}$. The first attempt to model the physics of these systems, accounting for the presence of neutral Hydrogen in the ISM, was done by Ref. 106, 107 extending the thin-layer approximation ${ }^{108}$ used to model cometary nebulae ${ }^{109,110,111}$. The thin layer approximation neglects 
the thickness of the nebula. However these models provide a good description of the shape and physical properties (thickness to hydrogen penetration, $\mathrm{H}_{\alpha}$ luminosity) in the head region. This was confirmed by more accurate 2-D axisymmetric simulations both in the hydrodynamical regime ${ }^{112,102}$ and in the relativistic MHD regime $^{113}$. A 3-D extension of the study of these systems to take into account either a non-uniform ambient medium, or the anisotropy in the pulsar wind energy flux (in analogy with 2-D simulations of Crab-like PWNe) has been presented by Ref. 114 .

Bow-shock models have also been recently developed and applied to the interaction and confinement of the pulsar wind in binary systems ${ }^{115,116,117}$. In this case it is the wind from the companion that provides the confining medium. These models have been used to explain orbital variations and modulations of the high energy comptonized emission.

\section{References}

1. R. A. Chevalier, Advances in Space Research 33, 456 (2004).

2. B. M. Gaensler and P. O. Slane, ARA\&SA 44, 17 (2006).

3. N. Bucciantini, Theory of Pulsar Wind Nebulae, in 40 Years of Pulsars: Millisecond Pulsars, Magnetars and More, eds. C. Bassa, Z. Wang, A. Cumming and V. M. Kaspi, American Institute of Physics Conference Series, Vol. 983 (2008).

4. K. W. Weiler and N. Panagia, A\&A 70, p. 419 (1978).

5. I. Asaoka and K. Koyama, 42, 625 (1990).

6. A. K. Harding, Spac.Scienc.Review 75, 257 (1996).

7. R. Bandiera, Properties of Nonthermal Emission in Plerions, in Highly Energetic Physical Processes and Mechanisms for Emission from Astrophysical Plasmas, eds. P. C. H. Martens, S. Tsuruta and M. A. Weber, IAU Symposium, Vol. 195May (2000).

8. O. Kargaltsev and G. G. Pavlov, X-ray Astronomy 2009; Present Status, MultiWavelength Approach and Future Perspectives 1248, 25 (2010).

9. J. J. Hester, $A R A \xi A$ 46, 127 (2008).

10. E. Amato and J. Arons, ApJ 653, 325 (2006).

11. L. Sironi and A. Spitkovsky, ApJ 726, p. 75 (2011).

12. L. Sironi and A. Spitkovsky, ApJ 698, 1523 (2009).

13. Y. E. Lyubarsky, MNRAS 345, 153 (2003).

14. Y. Lyubarsky and M. Liverts, ApJ 682, 1436 (2008).

15. L. Sironi and A. Spitkovsky, ApJ 741, p. 39 (2011).

16. P. Goldreich and W. H. Julian, ApJ 157, p. 869 (1969).

17. S. V. Bogovalov, $A \& A A$ 327, 662 (1997).

18. J. A. Hibschman and J. Arons, ApJ 560, 871(2001).

19. A. Barnes and J. D. Scargle, ApJ 184, 251(1973).

20. N. Bucciantini, J. Arons and E. Amato, MNRAS 410, 381 (2011).

21. A. M. Atoyan and F. A. Aharonian, MNRAS 278, 525 (1996).

22. S. Dall'Osso, G. Stratta, D. Guetta, S. Covino, G. De Cesare and L. Stella, A\&A 526, p. A121 (2011).

23. N. Bucciantini, E. Quataert, J. Arons, B. D. Metzger and T. A. Thompson, MNRAS 383, L25 (2008).

24. N. Bucciantini, E. Quataert, B. D. Metzger, T. A. Thompson, J. Arons and L. Del Zanna, MNRAS 396, 2038 (2009). 
25. N. Bucciantini, B. D. Metzger, T. A. Thompson and E. Quataert, MNRAS 419, 1537 (2012).

26. F. Pacini and M. Salvati, ApJ 186, 249 (1973).

27. S. P. Reynolds and R. A. Chevalier, ApJ 278, 630 (1984).

28. S. P. Reynolds and G. A. Chanan, ApJ 281, 673 (1984).

29. A. M. Atoyan, $A \xi A$ 346, L49 (1999).

30. W. Bednarek and M. Bartosik, AESA 405, 689 (2003).

31. D. F. Torres, A. N. Cillis and J. Martín Rodriguez, ApJL 763, p. L4 (2013).

32. S. J. Tanaka and F. Takahara, MNRAS 429, 2945 (2013).

33. S. J. Tanaka and F. Takahara, ApJ 715, 1248 (2010).

34. J. Martín, D. F. Torres and N. Rea, MNRAS 427, 415 (2012).

35. J. D. Gelfand, P. O. Slane and W. Zhang, ApJ 703, 2051 (2009).

36. M. J. Rees and J. E. Gunn, MNRAS 167, 1 (1974).

37. C. F. Kennel and F. V. Coroniti, ApJ 283, 694 (1984).

38. C. F. Kennel and F. V. Coroniti, ApJ 283, 710 (1984).

39. R. T. Emmering and R. A. Chevalier, ApJ 321, 334 (1987).

40. J. M. Blondin, R. A. Chevalier and D. M. Frierson, ApJ 563, 806 (2001).

41. E. van der Swaluw, A. Achterberg, Y. A. Gallant and G. Tóth, A\&A 380, 309 (2001)

42. R. A. Chevalier and C. Fransson, ApJ 395, 540 (1992).

43. N. Bucciantini, J. M. Blondin, L. Del Zanna and E. Amato, A $\& A$ 405, 617 (2003).

44. N. Bucciantini, R. Bandiera, J. M. Blondin, E. Amato and L. Del Zanna, A\& $A$ 422, 609 (2004)

45. O. C. de Jager, S. E. S. Ferreira and A. Djannati-Ataï, MHD and Radiation Modelling of G21.5-0.9, in American Institute of Physics Conference Series, eds. F. A. Aharonian, W. Hofmann and F. Rieger, American Institute of Physics Conference Series, Vol. 1085 (2008).

46. A. Melatos, MemSAIt 69, p. 1009 (1998).

47. F. C. Michel, ApJ 158, p. 727 (1969).

48. P. Goldreich and W. H. Julian, ApJ 160, p. 971 (1970).

49. V. S. Beskin, I. V. Kuznetsova and R. R. Rafikov, MNRAS 299, 341 (1998).

50. S. V. Bogovalov, A\&A 371, 1155 (2001).

51. T. Chiueh, Z.-Y. Li and M. C. Begelman, ApJ 505, 835 (1998).

52. E. Amato, M. Salvati, R. Bandiera, F. Pacini and L. Woltjer, A $8 A$ A59, 1107 (2000).

53. S. Reynolds, X-ray Spectral Structure in Pulsar-Wind Nebulae, in 35th COSPAR Scientific Assembly, ed. J.-P. Paillé, COSPAR Meeting, Vol. 352004.

54. J. J. Hester, P. A. Scowen, R. Sankrit, C. J. Burrows, J. S. Gallagher, III, J. A. Holtzman, A. Watson, J. T. Trauger, G. E. Ballester, S. Casertano, J. T. Clarke, D. Crisp, R. W. Evans, R. E. Griffiths, J. G. Hoessel, J. Krist, R. Lynds, J. R. Mould, E. J. O’Neil, Jr., K. R. Stapelfeldt and J. A. Westphal, ApJ 448, p. 240 (1995).

55. M. C. Weisskopf, J. J. Hester, A. F. Tennant, R. F. Elsner, N. S. Schulz, H. L. Marshall, M. Karovska, J. S. Nichols, D. A. Swartz, J. J. Kolodziejczak and S. L. O’Dell, ApJL 536, L81 (2000).

56. B. M. Gaensler, J. Arons, V. M. Kaspi, M. J. Pivovaroff, N. Kawai and K. Tamura, ApJ 569, 878 (2002).

57. F. J. Lu, Q. D. Wang, B. Aschenbach, P. Durouchoux and L. M. Song, ApJL 568, L49 (2002).

58. F. Camilo, B. M. Gaensler, E. V. Gotthelf, J. P. Halpern and R. N. Manchester, ApJ 616, 1118 (2004). 
59. G. G. Pavlov, M. A. Teter, O. Kargaltsev and D. Sanwal, ApJ 591, 1157 (2003).

60. R. W. Romani and C.-Y. Ng, ApJL 585, L41 (2003).

61. R. W. Romani, C.-Y. Ng, R. Dodson and W. Brisken, ApJ 631, 480 (2005).

62. P. Slane, D. J. Helfand, E. van der Swaluw and S. S. Murray, ApJ 616, 403 (2004).

63. M. C. Begelman and Z.-Y. Li, ApJ 397, 187 (1992).

64. S. S. Komissarov, MNRAS 303, 343 (1999).

65. L. Del Zanna, N. Bucciantini and P. Londrillo, A\&SA 400, 397 (2003).

66. E. van der Swaluw, A\&\&A 404, 939 (2003).

67. M. C. Begelman and Z.-Y. Li, ApJ 426, 269 (1994).

68. I. Contopoulos, D. Kazanas and C. Fendt, ApJ 511, 351 (1999).

69. A. N. Timokhin, MNRAS 368, 1055 (2006).

70. S. S. Komissarov, MNRAS 367, 19 (2006).

71. A. Spitkovsky, ApJL 648, L51 (2006).

72. N. Bucciantini, T. A. Thompson, J. Arons, E. Quataert and L. Del Zanna, MNRAS 368, 1717 (2006).

73. S. V. Bogovalov and D. V. Khangoulian, MNRAS 336, L53 (2002).

74. S. V. Bogovalov and D. V. Khangoulyan, Astronomy Letters 28, 373 (2002).

75. Y. E. Lyubarsky, MNRAS 329, L34 (2002).

76. S. S. Komissarov and Y. E. Lyubarsky, MNRAS 349, 779 (2004).

77. L. Del Zanna, E. Amato and N. Bucciantini, A\&A 421, 1063 (2004).

78. S. V. Bogovalov, V. M. Chechetkin, A. V. Koldoba and G. V. Ustyugova, MNRAS 358, 705 (2005).

79. L. Del Zanna, D. Volpi, E. Amato and N. Bucciantini, $A \mathscr{E} A$ 453, 621 (2006).

80. D. Volpi, L. Del Zanna, E. Amato and N. Bucciantini, A $\mathscr{G} A$ 485, 337 (2008).

81. N. Bucciantini, L. del Zanna, E. Amato and D. Volpi, A\&A 443, 519 (2005).

82. T. Schweizer, N. Bucciantini, W. Idec, K. Nilsson, A. Tennant, M. Weisskopf and R. Zanin, MNRAS 433, 3325 (2013).

83. N. Bucciantini and L. Del Zanna, A $\mathscr{J} A$ 454, 393 (2006).

84. N. F. Camus, S. S. Komissarov, N. Bucciantini and P. A. Hughes, MNRAS 400, 1241 (2009).

85. B.-I. Jun, ApJ 499, p. 282 (1998).

86. N. Bucciantini, E. Amato, R. Bandiera, J. M. Blondin and L. Del Zanna, A\& A 423, 253 (2004).

87. E. van der Swaluw, T. P. Downes and R. Keegan, A\& A 420, 937 (2004).

88. E. van der Swaluw, A. Achterberg and Y. A. Gallant, Rejuvenating the Shells of Supernova Remnants by Pulsar Winds, in Neutron Stars in Supernova Remnants, eds. P. O. Slane and B. M. Gaensler, Astronomical Society of the Pacific Conference Series, Vol. 2712002.

89. S. E. S. Ferreira and O. C. de Jager, $A \mathscr{G} A$ 478, 17 (2008).

90. M. C. Begelman, ApJ 493, p. 291 (1998).

91. K. Nalewajko and M. C. Begelman, MNRAS 427, 2480 (2012).

92. K. Mori, D. N. Burrows, G. G. Pavlov, J. J. Hester, S. Shibata and H. Tsunemi, Year-scale Morphological Variations of the X-ray Crab Nebula, in Young Neutron Stars and Their Environments, eds. F. Camilo and B. M. Gaensler, IAU Symposium, Vol. 2182004.

93. S. M. O'Neill, K. Beckwith and M. C. Begelman, MNRAS 422, 1436 (2012).

94. Y. Mizuno, Y. Lyubarsky, K.-I. Nishikawa and P. E. Hardee, ApJ 728, p. 90 (2011).

95. Y. Mizuno, Y. Lyubarsky, K.-I. Nishikawa and P. E. Hardee, ApJ 757, p. 16 (2012).

96. F. G. Smith, D. H. P. Jones, J. S. B. Dick and C. D. Pike, MNRAS 233, 305 (1988).

97. T. Velusamy, MNRAS 212, 359 (1985). 
98. M. F. Bietenholz, A Radio Study of the Crab Nebula., PhD thesis, UNIVERSITY OF TORONTO (CANADA).1991.

99. O. Porth, S. S. Komissarov and R. Keppens, MNRAS 431, L48 (2013).

100. J. M. Cordes, R. W. Romani and S. C. Lundgren, Nature 362, 133 (1993).

101. J. F. Bell, M. Bailes, R. N. Manchester, J. M. Weisberg and A. G. Lyne, ApJL 440, L81 (1995).

102. B. M. Gaensler, E. van der Swaluw, F. Camilo, V. M. Kaspi, F. K. Baganoff, F. YusefZadeh and R. N. Manchester, ApJ 616, 383 (2004).

103. G. G. Pavlov, D. Sanwal and V. E. Zavlin, ApJ 643, 1146 (2006).

104. O. Kargaltsev, Z. Misanovic, G. G. Pavlov, J. A. Wong and G. P. Garmire, ApJ 684, $542(2008)$.

105. C.-Y. Ng, N. Bucciantini, B. M. Gaensler, F. Camilo, S. Chatterjee and A. Bouchard, ApJ 746, p. 105 (2012).

106. N. Bucciantini and R. Bandiera, $A \& A$ A 375, 1032 (2001).

107. N. Bucciantini, $A \mathscr{E} A$ 393, 629 (2002).

108. J. L. Giuliani, Jr., ApJ 256, 624 (1982).

109. R. Bandiera, A\&A 276, p. 648 (1993).

110. F. P. Wilkin, ApJL 459, p. L31 (1996).

111. F. P. Wilkin, ApJ 532, 400 (2000).

112. N. Bucciantini, A\&A 387, 1066 (2002).

113. N. Bucciantini, E. Amato and L. Del Zanna, AESA 434, 189 (2005).

114. M. Vigelius, A. Melatos, S. Chatterjee, B. M. Gaensler and P. Ghavamian, MNRAS 374, 793 (2007).

115. S. V. Bogovalov, D. V. Khangulyan, A. V. Koldoba, G. V. Ustyugova and F. A. Aharonian, MNRAS 387, 63 (2008).

116. S. V. Bogovalov, D. Khangulyan, A. V. Koldoba, G. V. Ustyugova and F. A. Aharonian, MNRAS 419, 3426 (2012).

117. V. Bosch-Ramon, M. V. Barkov, D. Khangulyan and M. Perucho, A $\because A$ 544, p. A59 (2012). 